\title{
Simulation of OFDM based Software Defined Radio for FDD-LTE Uplink
}

\author{
Hansa Jha ${ }^{1}$, Pankaj M Gulhane ${ }^{2}$ \\ ${ }^{1}$ M. Tech Scholar, Electronics \& Telecommunication \\ ${ }^{2}$ Assistant Professor, Department of Electronics \& Telecommunication
}

\begin{abstract}
This paper gives an overview of Simulation of Software defined radio forLong Term Evolution (LTE) uplink based on Orthogonal Frequency division Multiplexing. Long Term Evolution is the outcome of the development of $3^{\text {rd }}$ generation partnership project (3GPP). This Evolution is a great boom in the mobile technologies towards 4th generation. As the Expected frequency range of LTE - 3GPP standard, it can accommodate an uplink speed of up to 50 Mbps and a downlink speed of up to 100 Mbps. In this paper, baseband section of Software defined radio is simulated for FDD based LTEuplinkdesign using MATLAB. Uplink design isformulatedusing SC-FDMA multiple access scheme with proposed methodology.A clipped Selective mapping technique is used in conjunction with Root raised cosine pulse shaping to reduce the PAPR content of SCFDMA which is a main disadvantage of OFDM systems.
\end{abstract}

Keywords: SDRLTE, 3GPP, 4G, SC-FDMA, OFDM, CSLM, RRC

\section{Introduction of Software Defined Radio}

Software defined Radio (SDR) is an architecture fully applicable to many of the communication standards. Software radio may be interpreted as the shift from digital radio to multi-band multi-mode software radio. The SDR forum, in collaboration with the Institute of Electrical \& Electronic Engineers (IEEE) group, established a definition of SDR that explains consistency and a clear picture of the advancements in the technology and associated benefits. Thus SDR can be defined as "A Radio in which the function of some or entire physical layer are made to be software defined" [1].

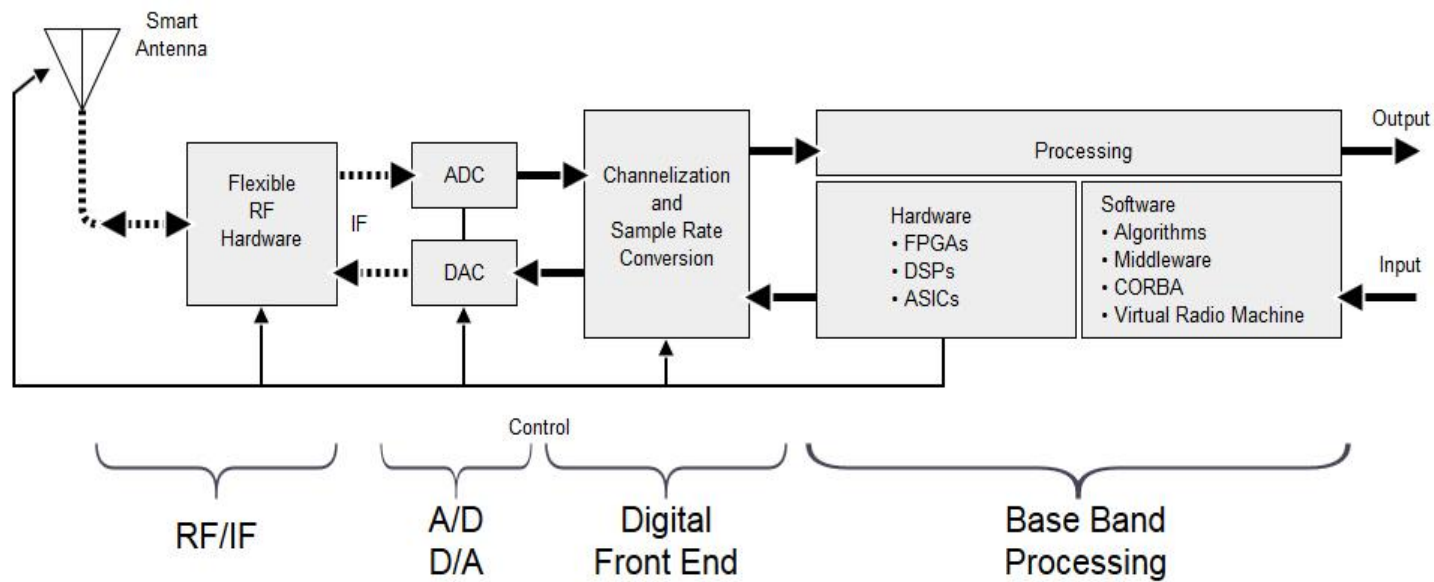

Figure 1: Software Defined Radio architecture [1]

This concept of SDR allows the communication systems to use minimum hardware resources with maximum adaptability for the different standards. Different software are available for the programmability of the software defined radios like MATLAB, SIMULINK and high level languages like $\mathrm{C}$ etc.
A software defined radio is a platform of communication whose functionality defined in software with the aid of hardware components. The efficiency towards the use of software removes the additional expenses on the hardware of SDR. In order to maintain inter portability, the radio systems might be developed in a well-defined manner with specific architectures [2.]Software Defined Radio is based on the technology in which a single hardware part can accommodate large number of frequencies over a particular bandwidth and process these in the software.

\section{Orthogonal \\ Frequency \\ Division Multiplexing}

OFDM is the abbreviation for Orthogonal Frequency Division Multiplexing. The basic principle of OFDM defines sub carrier mapping which needs large number of closely spaced sub carrier signals which are orthogonal to each other. These are used to carry the original data on number of parallel data channels. Thus OFDM can be regarded as an extension of single carrier modulation to the 


\section{International Journal of Science and Research (IJSR) \\ ISSN (Online): 2319-7064}

Index Copernicus Value (2013): 6.14 | Impact Factor (2015): 6.391

multicarrier modulation scheme, where a single data channel is sent over a number of lower data rate orthogonal subcarriers. To maintain the orthogonality criteria of subcarriers, OFDM system employs Inverse fast Fourier transform (IFFT) and Fast Fourier Transform (FFT) to generate the orthogonal frequency.

The first OFDM transmission was started in 1950-60 for the parallel transmission. In 1999, OFDM proved quite helpful for the higher bit rate systems. Recently various VLSI standards are using the OFDM for implementation of cheap blocks for IFFT and FFT operations. OFDM allows the selection of operating frequencies on the basis of the preserved orthogonality of signals [3]. Figure 2 shows a block diagram of Orthogonal Frequency division Multiplexing.

Input is generated by any of the binary sequence generator, is known as baseband signal. A number of digital modulation schemes can be used for the modulation of input. Use of Different modulation schemes providesdifferent constellation diagram.

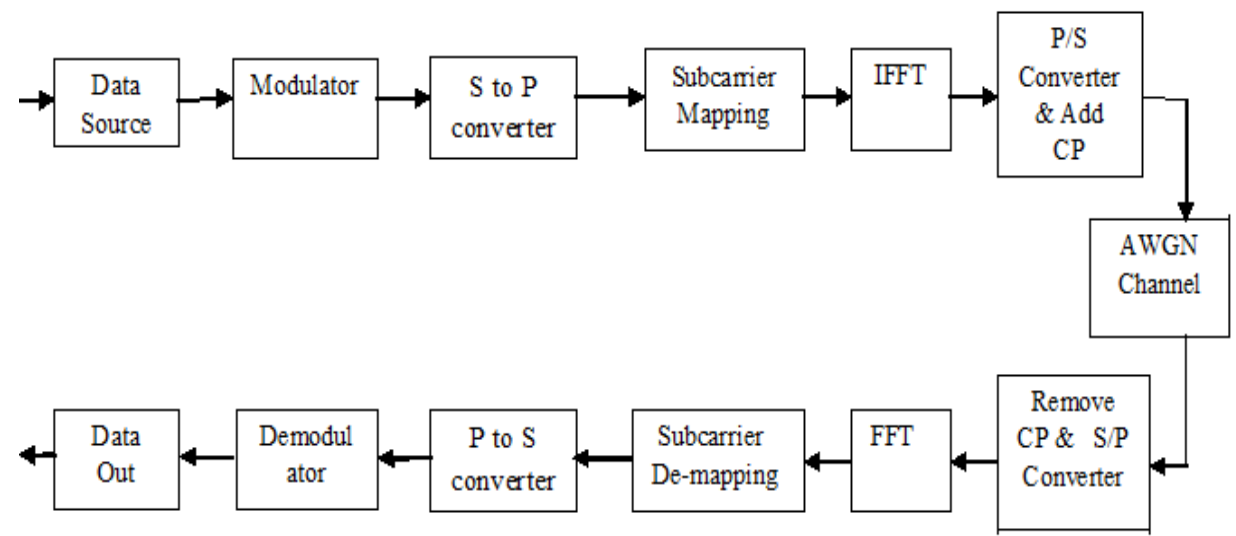

Figure 2: Block Diagram ofOrthogonal Frequency Division Multiplexing (OFDM)

Anothervariation of OFDM uses some distinct modulation methods based on sub-carrier mapping. A serial to parallel converter converts the incoming serial data into $\mathrm{N}$ - parallel streams.These generated parallel streams individually modulate each sub-carriers through the Inverse Fast Fourier Transform (IFFT) block. This is the most important fundamental of OFDM Systems. The two important hurdles in the OFDM are Inter symbol Interference (ISI) and Inter block Interference (IBI), which reduces or degrades the performance of communication standards. To eliminate the effects of ISI and IBI on the OFDM signals, cyclic Prefix are used.Figure 3 shows a time domain representation of orthogonal Frequency division multiplexing (OFDM).

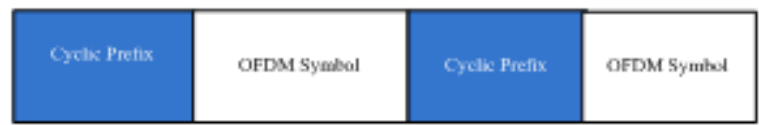

Figure 3: Time domain Representation of OFDM

Figure 4shows the frequency domain representation of orthogonal frequency division multiplexing (OFDM) signal. In the spectrum, each of the Sinc function corresponds to a sinusoidal carrier gets modulated by a rectangular waveform which contains the original information symbol.

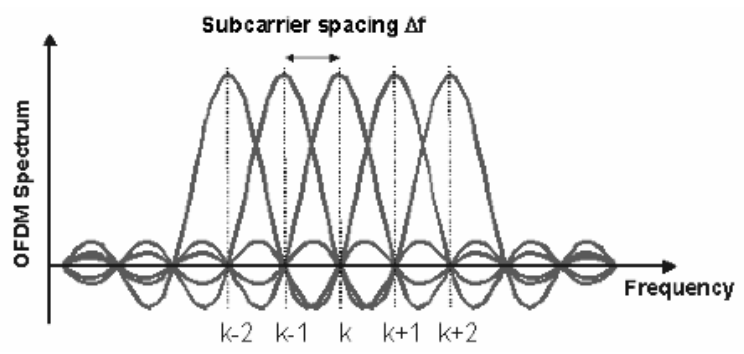

Figure 4: Frequency Domain Representation of OFDM
OFDM became a new fundamental aspect for the IEEE 802.1n standards for numerous applications like WLAN, WIMAX, HIPERLAN, LTE-3GPP etc.

Table 1: Advantage and Disadvantages of OFDM

\begin{tabular}{|c|c|}
\hline $\begin{array}{c}\text { Channel estimation and Channel } \\
\text { of the system. }\end{array}$ & $\begin{array}{c}\text { A main demerit of OFDM is } \\
\text { higher Peak to Average Power } \\
\text { Ratio (PAPR). }\end{array}$ \\
\hline $\begin{array}{c}\text { OFDM allows full use of the } \\
\text { frequency spectrum by } \\
\text { overlapping }\end{array}$ & $\begin{array}{c}\text { Cyclic prefix cause the loss of } \\
\text { efficiency of the OFDM systems. }\end{array}$ \\
\hline $\begin{array}{c}\text { Reduction of Inter Symbol } \\
\text { with the use of cyclic prefix } \\
\text { withe and multipath fading }\end{array}$ & $\begin{array}{c}\text { A complex transmitter circuit and } \\
\text { high power amplifiers are } \\
\text { Required }\end{array}$ \\
\hline $\begin{array}{c}\text { Channel Equalization becomes } \\
\text { simpler with the OFDM. }\end{array}$ & $\begin{array}{c}\text { Carrier frequency offset and drift } \\
\text { causes the degradation the } \\
\text { OFDM signals. }\end{array}$ \\
\hline $\begin{array}{c}\text { Easy modulation and } \\
\text { demodulation with the use of } \\
\text { IFFT and FFT blocks. }\end{array}$ & \\
\hline $\begin{array}{c}\text { More resistive to the frequency } \\
\text { selective fading compared to } \\
\text { single carrier systems. }\end{array}$ & \\
\hline
\end{tabular}

\section{Long Term Evolution (LTE)}

Long Term Evolution (LTE) is the next step in mobile technology towards $4^{\text {th }}$ generation advancement. Expected in earlier time, LTE is a 3 GPP standard that provide an uplink transmission speed of up to $50 \mathrm{Mbps}$ and a downlink reception speed of up to $100 \mathrm{Mbps}$. Bandwidth of LTEs are scalable from $1.25 \mathrm{MHz}$ to $20 \mathrm{MHz}$. This will fulfill the requirements of different network operations that have different bandwidth allocations and also allow different services to the operators depending upon the spectrum. LTE also provides improved spectral efficiency in $3 \mathrm{G}$ and $4 \mathrm{G}$

\section{Volume 5 Issue 6, June 2016}




\section{International Journal of Science and Research (IJSR) \\ ISSN (Online): 2319-7064}

Index Copernicus Value (2013): 6.14 | Impact Factor (2015): 6.391

networks allowing carries to provide more data and voice services over a given bandwidth. It is designed to meet carrier requirement for high speed data and media transport as well as high capacity voice support for multimedia unicast and multimedia broadcast services [4].

Long term evolution (LTE) development includes higher data rates and lower latencies. Basically Physical layer design is used for any of the mobile standard. LTE standard defines multimode air interface based on orthogonal Frequency Division Multiplexing (OFDM).Many Methods employed in LTE are relatively new in Cellular applications like OFDM, OFDMA, MIMO-OFDM and SC-FDMA.

LTE represents a significant advance in cellular technology and it defines are placement high speed radio access technique for top speed information and media transport in addition as high capability voice support at interval succeeding decade. LTE defines Long Term evolution routes for UMTS network operators for fourth generation mobile networks. In recent years it supports GSM (global System for Mobile Communication), GPRS (General packet Radio service), and EDGE in addition as WCDMA (wideband code division multiple access) and currently HSPA[4].
LTE Uplink requirements differ from downlink requirements in several ways. Power consumption is a key point in LTE transceiver design. Also high PAPR and efficiency loss associated with OFDM signaling are of major considerations in designing. Thus an alternative to this problem in OFDM is used in the LTE Uplink. Basically LTE is based on the principal of orthogonal frequency Division Multiplexing with the required frequency synchronization. It supports both kind of duplex modes frequency division duplex (FDD) \& Time division Duplex (TDD).

\subsection{LTE Physical Layer Mapping}

\subsubsection{LTE FDD Frame Structure}

LTE FDD frame Structure stands for Frequency Division Duplex mode that typically works on the half and full duplex modes each. Figure shows LTE frame structure supported FDD mode. It incorporate total 20 slots numbered 0 to 19 ,each having equal length of $0.5 \mathrm{~ms}$. A radio frame incorporates sub-frame, each have 2 slots therefore total of 10 sub-frames exist.

Table 2: Long Term Evolution Specifications

\begin{tabular}{|c|c|c|}
\hline SN & LTE Parameters & Target / specifications \\
\hline 1 & Channel Bandwidth & $1,3,4,5,10,20 \mathrm{MHz}$ \\
\hline 2 & System Mode & TDD,FDD half FDD Modes \\
\hline 3 & Data type & ALL packet Switched voice \& Data. No Circuit switched \\
\hline 4 & Access Techniques & SC-FDMA (Uplink), OFDMA (Downlink) \\
\hline 5 & Modulation Schemes & BPSK, QPSK, M- QAM \\
\hline 6 & Antenna Requirement & 50 Mbps(QPSK), 57 Mbps(16 QAM) \\
\hline 7 & Peak Uplink Speed & Upto 100 Mbps \\
\hline 8 & Peak Downlink Speed & Full Performance upto 5 Km \\
\hline 9 & Coverage & 5 bps or higher for Downlink \\
& Peak Spectral Efficiency & CP latency $<100$ ms , UE latency $<50 \mathrm{~ms}$ \\
\hline 10 & & \\
\hline 11 & Latency & \\
\hline
\end{tabular}

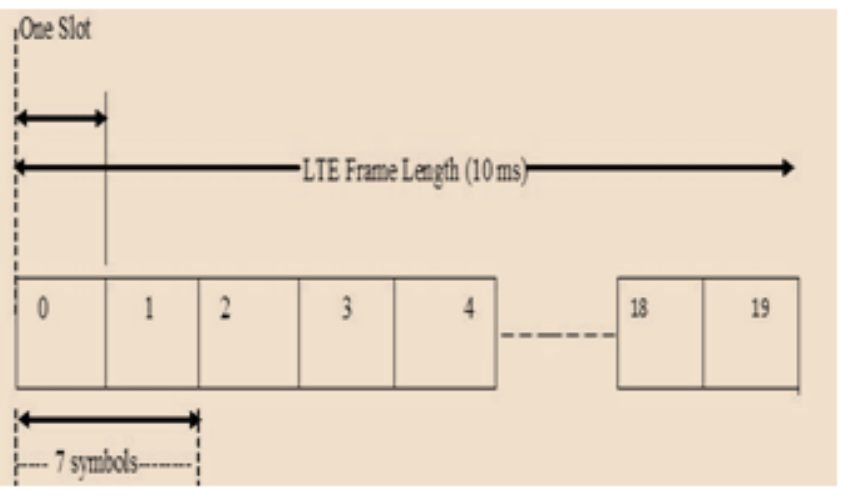

Figure 5: LTE FDD Frame Structure

\subsubsection{LTE TDD Frame Structure}

TDD based LTE Frame is split into 2 equal sub frames every of $5 \mathrm{~ms}$ length. Each of the frames are once more divided into five sub-frames every of $1 \mathrm{~ms}$ time length. Figure Shows layout of LTE Frame structure base on time division duplex Mode (TDD).A single radio frame of 10 ms length incorporates 2 slots of $5 \mathrm{~ms}$ every. Within the slotted sub frames, 3 fields particularly guard amount, transmission Pilot time slot (UpPTS) \& downlink pilottimeslot (DwPTS) exist. These fields are configurable for different transmission downlink configurations.

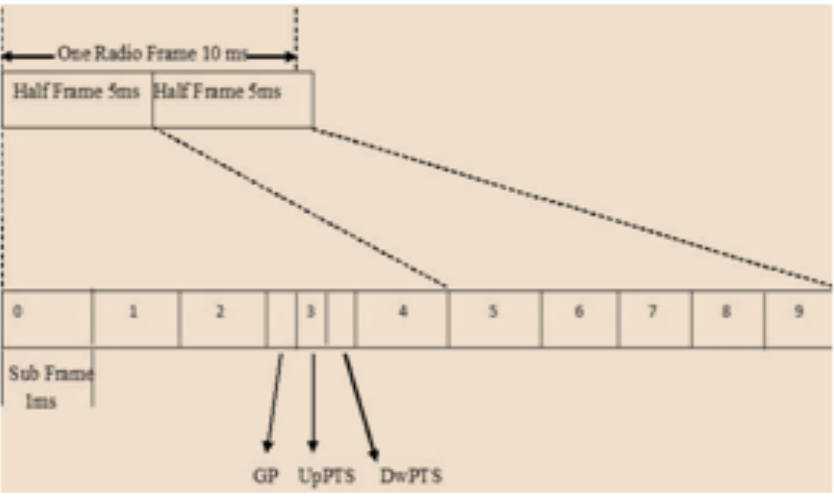

Figure 6: LTE TDD Frame Structure

\section{Volume 5 Issue 6, June 2016 www.ijsr.net}




\section{PAPR Problem in OFDM}

PAPR of OFDM signal is defined as the ratio of maximum peak power to its average power.OFDM signal is the vector sum of a number of subcarriers with different phases..Resultant peak value of an OFDM signal become substantially larger than average value.The expression for the PAPR for any of the OFDM signal is represented by

$$
\mathrm{PAPR}=\frac{\max 0 \leq t<N T|x(t)|^{2}}{\frac{1}{N T} \int_{0}^{N T}|x(t)|^{2} d t}
$$

Nowadays Complementary Cumulative Distribution factor (CCDF) have been replaced the use of CDF since it can provide the better measurement of probability of PAPR threshold of data streams. So the probability of PAPR threshold measurement can be represented in terms of $\mathrm{CCDF}$ equals to $\mathrm{P}(\mathrm{PAPR}>\mathrm{z})=1-\mathrm{P}(\mathrm{PAPR} \leq z)$

The only disadvantage of the PAPR reduction methods is that this method introduces a substantial complexity in the implementation of transmitter and receiver.

\section{Methodology}

This paper explains the implementation and simulation of Software Defined Radio for uplink LTE. It uses Single Carrier frequency division multiple access scheme for implementation of Uplink transmission for LTE standards. Since PAPR is a major challenge in the performance of LTE systems. Reduction of PAPR is an important constraint in the design of LTE systems. The advantage of higher data rates of LTE systems reduces if it has high PAPR content. Thus a technique Clipped SLM is introduced in conventional SCFDMA LTE to reduce the PAPR level to improve performance.

\subsection{ProposedSCFDMA with Clipped SLM with RRC Pulse Shaping}

Uplink design for a LTE transmitter is realized by using DFT spread Frequency division multiple accesses also called (SC- FDMA). SC-FDMA is defined as a hybrid modulation scheme that provides low Peak to average Power (PAPR) of OFDM.In a SC-FDMA scheme, the symbol length is made to be equal as the occupied bandwidth. LTE uses $15 \mathrm{KHz}$ subcarriers having spacing of 66.7 microseconds. These 15 $\mathrm{KHz}$ Subcarriers provide a high data rate of in the $20 \mathrm{MHz}$ bandwidth.

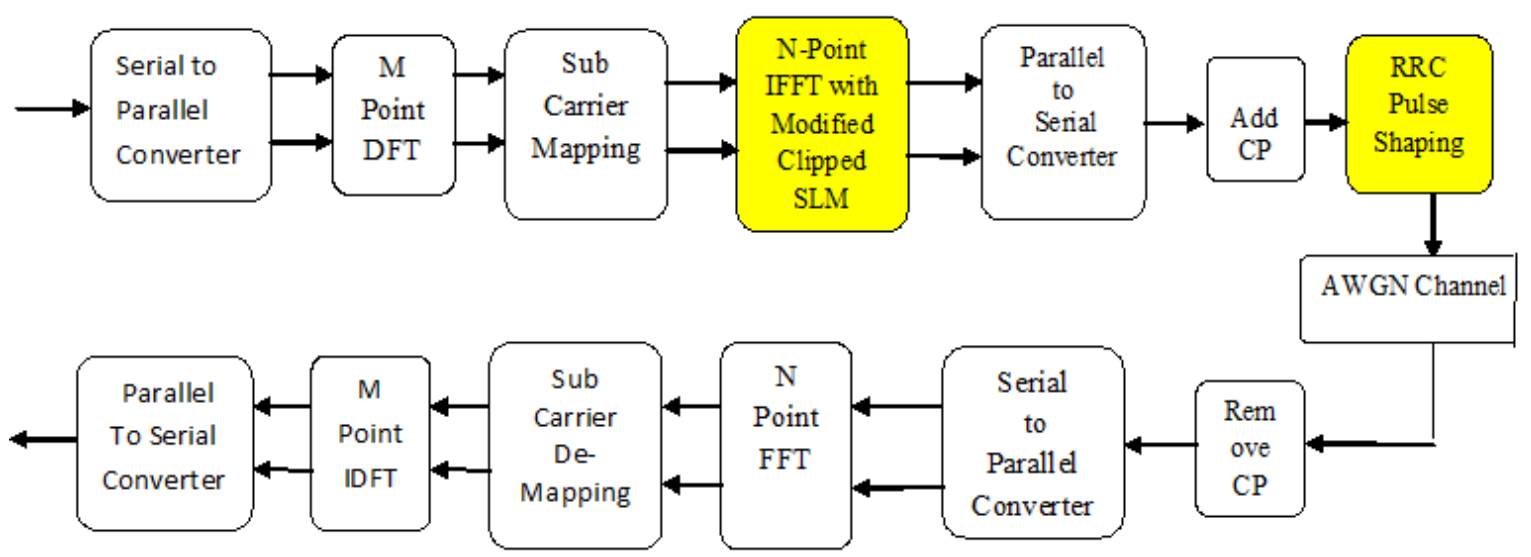

Figure7: Proposed Methodology for Software Defined Radio for FDD- LTEUplink

\subsection{Clipped Selected Mapping(CSLM) with RRC pulse shaping}

In Clipped Selective mapping method of PAPR reduction in orthogonal frequency division multiplexing, a set of data blocks are generated which contain actual information to be transmitted. Among the different generated blocks, the most favorable block with minimum PAPR is referred for the transmission. These generated data blocks consist of vectored Ncomplex symbols. Out of $\mathrm{N}$ symbols, individual one represents a modulation symbol.

Figure shows a block diagram of Selective Mapping (SLM) method for PAPR reduction. Each data block is multiplied by $\mathrm{V}$ different phase vector of length $\mathrm{N} B \mathrm{v}=\left[\mathrm{b}_{0} \mathrm{v}, 0\right.$, $\left.\mathrm{b}_{\mathrm{v}}, 1, \ldots . . \mathrm{bu}, \mathrm{N}-1\right]^{\mathrm{T}}(\mathrm{v}=0,1, \ldots . \mathrm{V}-1)$, resulting in $\mathrm{V}$ different data blocks. Thus $\mathrm{V}^{\text {th }}$ phase sequence after multiplication is

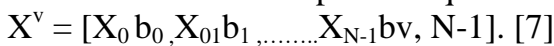

Accordingly, each multiplied phase sequence is passed through individual IFFT blocks, so require $\mathrm{V}$ number of IFFT operations. These IFFT blocks with binary sequence might reduce the complexity as compared to IDFT blocks. Instead of choosing a sequence of lowest PAPR content among these IFFT block output, a pairing method is used. Iterative PAPR selection is performed by combining a pair of output of IFFT blocks into a sub-block and then choosing one with low PAPR of incoming sequences. Before the final selection of PAPR content, output of each sub-block is then passed through amplitude clipping. This clipping of already low PAPR signal tends to reduce PAPR level. After that again a selection is performed for clipped Sequences of resulting Sub-blocks with previously selected low PAPR content. It is possible to implement SLM techniques do no need side information to be transmitted to the receiver end. This method does not send side information to receiver. 


\section{International Journal of Science and Research (IJSR) \\ ISSN (Online): 2319-7064}

Index Copernicus Value (2013): 6.14 | Impact Factor (2015): 6.391

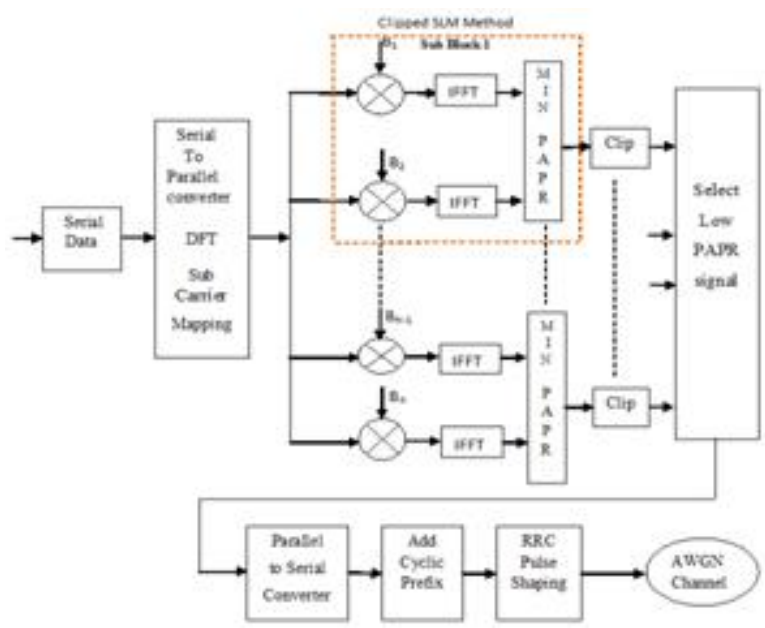

Figure 8: Clipped Selective Mapping with Root Raised Cosine Pulse Shaping method

A Root Raised Cosine (RRC) filter is used in addition with CSLM to shape the low PAPR signal. This RRc Pulse shaping method also provide reduction in peak value of Signal.

\section{Simulation Result}

Figure 9 shows a BER vs SNR comparison of SCFDMA, with SLM, with proposed Clipped SLM and CSLM with RRC pulse shaping method for LTE Software Defined Radio.

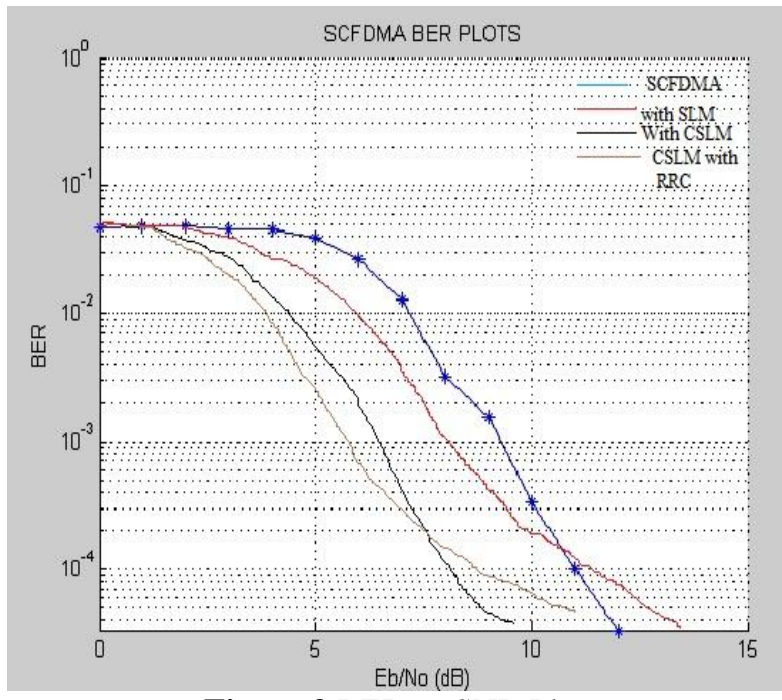

Figure 9 BER vs SNR Plot

Figure 9 shows a PAPR comparison of SCFDMA, with SLM, with proposed Clipped SLM and CSLM with RRC pulse shaping method for LTE Software Defined Radio.

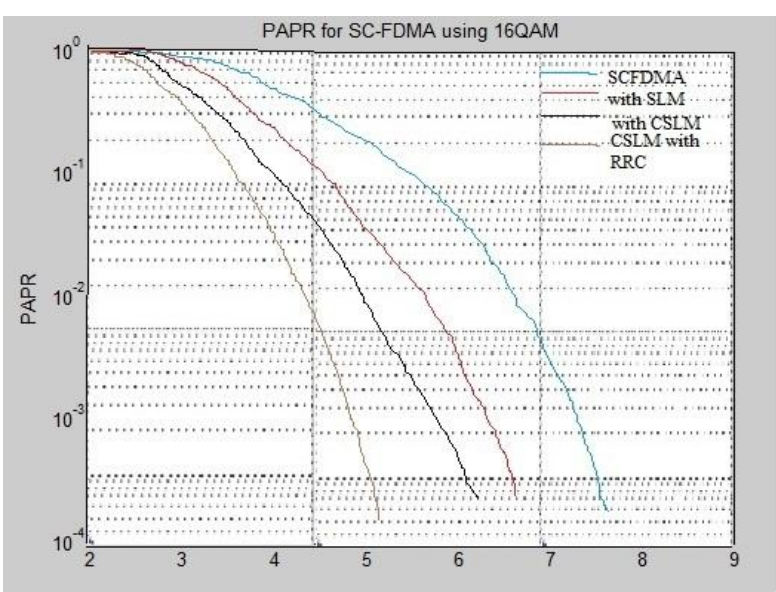

Figure10: CCDF plot for PAPR

\section{Conclusion}

Software Defined Radio based on FDD frame structure for LTE uplink is simulated in this work. LTE uplink is realized by Single carrier frequency division multiple access (SCFDMA). A methodology clipped Selective Mapping (CSLM) is proposed to reduce the peak power in SCFDMA signal followed by RRC filter. Obtained Results Show that Clipped SLM with RRC pulse shaping gives a better improvement in PAPR reduction in comparison with conventional SLM PAPR reduction in SCFDMA-LTE uplink

\section{Future Work}

More sophisticated and enhanced software defined radio can be developed with the implementation of such techniques which might be helpful towards the up-gradation of different enhancement in their battery usage aspects especially in case of mobile terminals or base wireless standards. A clustered SCFDMA with such CSLM PAPR reduction method can be implemented that can provide speed more than 100 mbps and more battery usage can be achieved. This can also be implemented for LTE downlink with OFDMA which has problem of high PAPR content.

\section{References}

[1] Shriram K Vasudevan and et al. 2010 "SoftwareDefined Radio Implementation (With simulation \& analysis)" International Journal ofComputer Applications, Volume 4, No.8, (0975 8887.

[2] Mehul R. Naik and C. H. Vithalani .2013. "The Software-Defined Radio is now a reality" International Journal of Advanced Research in Electrical, Electronics and Instrumentation Engineering, Vol. 2,Issue 7.

[3] Hazim Salah Abdulsatar. 2013. "Simulation and Implementation of Orthogonal Frequency Division Multiplexing (OFDM) Model Based SDR", International Journal of Engineering and Advanced Technology (IJEAT, Volume-3, Issue-2, ISSN: 22498958.

[4] M.A. Mohamedand et al.2014."Performance Analysis of LTE Physical Layer Based on 3GPP Release- 8",

Volume 5 Issue 6, June 2016 www.ijsr.net 


\section{International Journal of Science and Research (IJSR) \\ ISSN (Online): 2319-7064}

Index Copernicus Value (2013): 6.14 | Impact Factor (2015): 6.391

IJCSI International Journal of Computer Science Issues, Vol. 11, Issue 4, No 2, ISSN (Print): 1694-0814.

[5] Cristina Ciochina and Hikmet Sari. 2010. A Review of OFDMA and Single-Carrier FDMA and Some Recent Results" Advances In Electronics And Telecommunications, Vol. 1,

[6] IshanGirdhar and et al.2013."Performance Analysis of DFT Spread OFDM Systems" International Journal of Advances in Computer Science and Technology, Volume 2, No.5.

[7] Houshou Chen and Hsinying Liang, "A Modified Selective Mapping with PAPR Reduction and Error Correction in OFDM Systems" WCNC 2007 proceedings.

[8] LTE in a Nutshell: The Physical Layer, White Paper 2010.

[9] Vulnerabilities of LTE and LTE-Advanced Communication; White Paper

[10]3GPP LTE: Introducing Single-Carrier FDMA

[11] OFDMA vs. SC-FDMA,Magazine Published in IEEE Wireless Communications

\section{Author Profile}

Hansa Jha, M. Tech scholar in Electronics and TelecommunicationEngineering from Disha Institute of Management and Technology, Raipur.

Prof. Pankaj M. Gulhane received the B.E. and M.E. degrees in Electronicsand Telecommunication Engineering from Amravati University, India,in 2000 and 2007, respectively. He joined Electronics and Telecommunication Engineering Department of Disha Institute of Management and Technology, as an Assistant Professor in October 2007. His currentresearch interests are in the areas of Space Time Coding, Cooperative Communications and MIMO-OFDM system. 